\title{
Cross-Modal Subspace Clustering via Deep Canonical Correlation Analysis
}

\author{
Quanxue Gao, ${ }^{1}$ Huanhuan Lian, ${ }^{1 *}$ Qianqian Wang, ${ }^{1 \dagger}$ Gan Sun ${ }^{2}$ \\ ${ }^{1}$ State Key Laboratory of Integrated Services Networks, Xidian University, Xi' an 710071, China. \\ ${ }^{2}$ State Key Laboratory of Robotics, Shenyang Institute of Automation, Chinese Academy of Sciences, China. \\ qxgao@xidian.edu.cn, lianhh1212@163.com, qianqian174@foxmail.com, sungan1412@gmail.com
}

\begin{abstract}
For cross-modal subspace clustering, the key point is how to exploit the correlation information between cross-modal data. However, most hierarchical and structural correlation information among cross-modal data cannot be well exploited due to its high-dimensional non-linear property. To tackle this problem, in this paper, we propose an unsupervised framework named Cross-Modal Subspace Clustering via Deep Canonical Correlation Analysis (CMSC-DCCA), which incorporates the correlation constraint with a self-expressive layer to make full use of information among the inter-modal data and the intra-modal data. More specifically, the proposed model consists of three components: 1) deep canonical correlation analysis (Deep CCA) model; 2) self-expressive layer; 3) Deep CCA decoders. The Deep CCA model consists of convolutional encoders and correlation constraint. Convolutional encoders are used to obtain the latent representations of cross-modal data, while adding the correlation constraint for the latent representations can make full use of the information of the inter-modal data. Furthermore, self-expressive layer works on latent representations and constrain it perform self-expression properties, which makes the shared coefficient matrix could capture the hierarchical intra-modal correlations of each modality. Then Deep CCA decoders reconstruct data to ensure that the encoded features can preserve the structure of the original data. Experimental results on several real-world datasets demonstrate the proposed method outperforms the state-of-the-art methods.
\end{abstract}

\section{Introduction}

In machine learning, classification (Liu, Tsang, and Müller 2017; Liu and Tsang 2017) and clustering are two core tasks. However, clustering task has attracted considerable attention due to the fact that label information is difficult to obtain in real applications. Most traditional clustering methods mainly focus on the clustering problem of lowdimensional data. Generally, traditional clustering methods can be roughly divided into five categories: 1) Non-negative matrix factorization (NMF) clustering (Akata, Thurau, and

\footnotetext{
${ }^{*}$ Corresponding author: Huanhuan Lian

${ }^{\dagger}$ Corresponding author: Qianqian Wang Copyright (c) 2020, Association for the Advancement of Artificial Intelligence (www.aaai.org). All rights reserved.
}

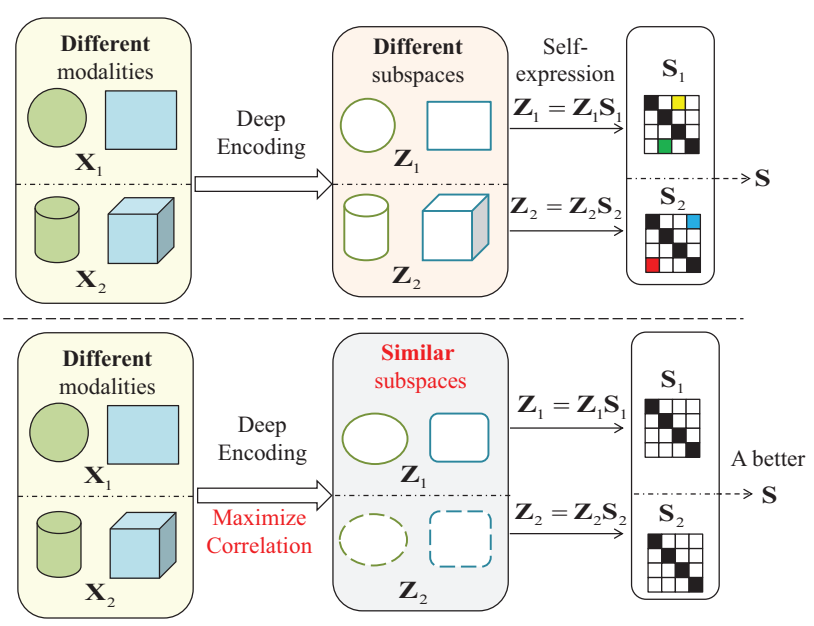

Figure 1: The illustration shows the influence of correlation constraint. The learned features of two modalities in the subspace are quite different without the correlation constraint, which resulting a bad shared coefficient matrix $\mathbf{S}$, because it's too difficult to reflect the different structure of two modalities simultaneously. However, combining the correlation constraint with a self-expressive layer, we can learn a better shared coefficient matrix $\mathbf{S}$. $\mathbf{X}_{\mathbf{1}}$ and $\mathbf{X}_{\mathbf{2}}$ are the input data. $\mathbf{Z}_{1}$ and $\mathbf{Z}_{\mathbf{2}}$ are the latent representations in subspace. $\mathbf{S}_{\mathbf{1}}$ and $\mathbf{S}_{\mathbf{2}}$ are the self-expression coefficient matrices.

Bauckhage 2011); 2) Multi-kernel learning strategy (Guo et al. 2014); 3) Subspace clustering methods (Chaudhuri et al. 2009); 4) Self-representation based methods (Yin et al. 2015); 5) Graph constraint based methods (Xia et al. 2014; Nie, Cai, and Li 2017). Among these above methods, subspace and self-representation based methods have received a lot of attention and achieved remarkable results. However, these traditional methods adopt shallow and linear embedding functions to reveal the intrinsic structure of data, which cannot simulate the high-dimensional nonlinear characteristics of data very well. Especially, for subspace clustering methods, high-dimensional data can easily lead to "curse of dimensionality". 
To solve the "curse of dimensionality" problem, Elhamifar et al. (2013) propose a Sparse Subspace Clustering (SSC) method to reduce dimension. Patel et al. (2015) propose Latent Space Sparse Subspace Clustering (LSSC) that simultaneously performs dimensionality reduction and sparse coding for SSC. With the development of neural networks, some people introduce the deep learning to solve subspace clustering problems (Ji et al. 2017; Sun et al. 2018; Li et al. 2019). For instance, Ji et al. (2017) use stacked auto-encoders as their basic model and adopt self-expression to learn the affinity of data in latent space for clustering. Deep Multi-modal Subspace Clustering (DMSC) (Abavisani and Patel 2018) presents a convolutional neural network (CNN) approach for unsupervised multi-modal subspace clustering. However, for cross-modal (Rasiwasia et al. 2010; Jia, Salzmann, and Darrell 2011) subspace clustering, there are only a few superior methods. For example, Zhang et al. (2007) propose to exploit cross-modal correlations to cluster two modalities data. He et al. (2015) use crossmodal learning via the pairwise constraint and aim to find the common hidden structure of cross-modal data. These cross-modal methods reduce the semantic gap of the intermodal data and improve the clustering accuracy. However, they cannot well capture nonlinear feature for cross-modal clustering. Moreover, cross-modal subspace clustering still confronts the following challenges:

- How should we consider the correlations of the intermodal data and the intra-modal data simultaneously to learn a representative shared subspace representation to improve clustering accuracy?

- How could we guarantee that the features encoded by the deep network can still reflect the structural distribution of the original data?

To overcome these challenges, we propose a novel CrossModal Subspace Clustering framework via Deep Canonical Correlation Analysis (CMSC-DCCA) to improve clustering performance. The proposed method consists of three parts: deep canonical correlation analysis (Deep CCA) (Andrew et al. 2013) model, a self-expressive layer and Deep CCA decoders. In the Deep CCA model, we map the highdimensional nonlinear cross-modal data into latent representations by deep convolution encoders; meanwhile, correlation constraint can maximize the correlations of the intermodal data. For the self-expressive layer, a self-expressive loss function is employed for the latent representation, which can reveal the correlation information among intramodal data. In addition, we add the Deep CCA decoders to reconstruct the original data, which ensures the representations processed by the encoders can still well reflect the characteristics of the original data. Figure 1 shows the purpose of combining the correlation constraint with a self-expression layer. Intuitively, without the correlation constraint, it is difficult to learn a shared subspace representation that reflects the structure of two modalities data simultaneously. The main contributions of our method are summarized as:

- We propose a novel deep cross-modal subspace clustering method (CMSC-DCCA) by incorporating the correlation constraint with a self-expression layer, which can consider both the correlations of the inter-modal data and the distribution of the intra-modal data.

- We add Deep CCA decoders into our model to reconstruct data, which ensures that the encoded features can well reflect the overall structural distribution of original data.

- We give an efficient algorithm to optimize loss function and train the entire network at once to reduce the calculation of parameters. In addition, we perform spectral clustering on shared coefficient matrix. Experiments show our model achieves the best clustering performance.

\section{Related work}

In real life, data are often described by different modalities. Therefore, cross-modal data processing is getting more attention, which focuses on maximizing the correlations of the inter-modal data. In this section, we provide a brief review about the correlation studies on cross-modal data.

Among cross-modal data correlation studies, the most representative method is based on Canonical Correlation Analysis (CCA) (Kim, Kittler, and Cipolla 2007). The main idea of CCA is to find the mapping vector of each modality in the subspace by maximizing the correlations of the inter-modal data. However, CCA can only calculate the linear correlations of the inter-modal data. In real applications, the relationships between cross-modal data may be nonlinear. To solve this problem, some nonlinear CCA methods have been proposed, such as, the Kernel Canonical Correlation Analysis (KCCA) (Akaho 2006) and Locality Preserving CCA (LPCCA) (Sun and Chen 2007). However, these methods have high computational complexity. In addition, it is easy overfitting and relatively difficult to choose a suitable kernel function for KCCA method. Deep Canonical Correlation Analysis (Deep CCA) (Andrew et al. 2013) method can learn complex nonlinear transformations of the inter-modal data such that the learned representations are highly correlated through a multi-layer deep network. The disadvantage of Deep CCA is that it cannot reconstruct data and further results in poor clustering performance. Wang et al. (2016) further propose Deep Canonically Correlated Auto-encoders (DCCAE) with an autoencoder to reconstruct data. Deep Generalized Canonical Correlation Analysis (DGCCA) (Benton et al. 2019) learns a modal-independent representation to cluster, which reduces redundant information of data. However, both DCCAE and DGCCA methods do not consider the correlations of the intra-modal data.

\section{The Proposed Method}

In this section, we first give the motivations of Cross-Modal Subspace Clustering via Deep Canonical Correlation Analysis model. Then we introduce the framework of the proposed model. Finally we analyze the loss functions and training process in detail.

\section{Motivations}

According to previous related work, there are two motivations for our method as follows: 


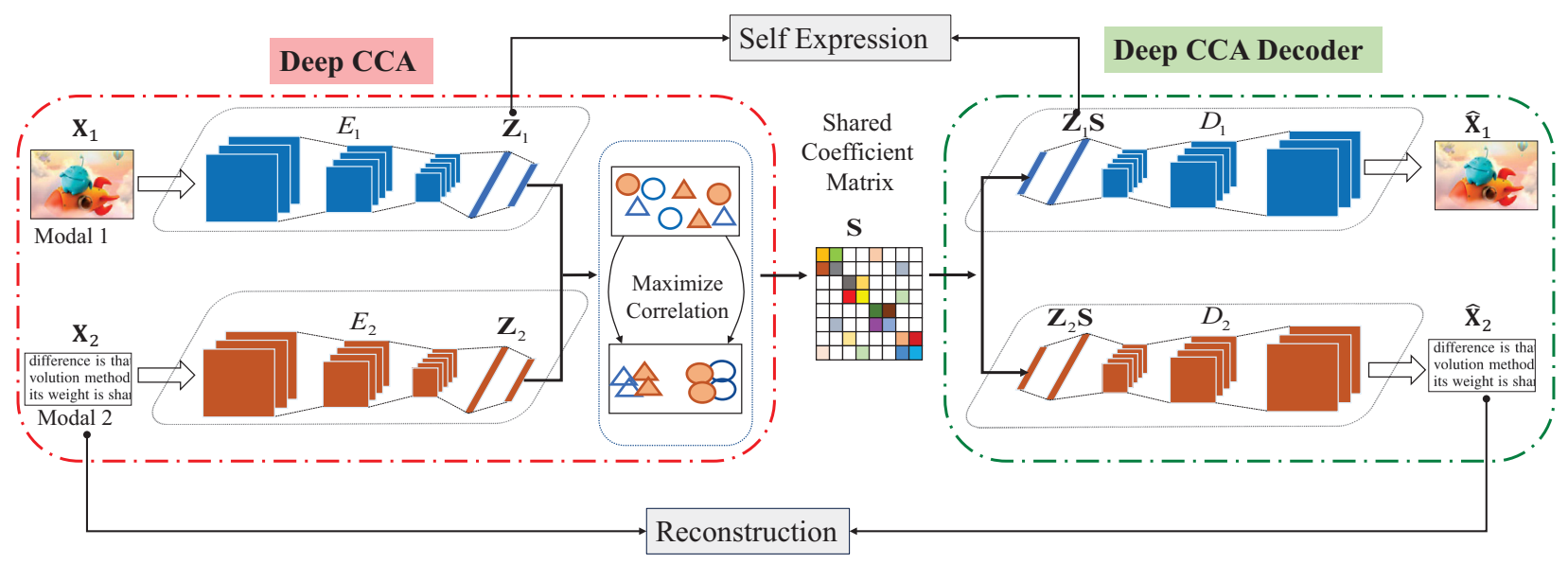

Figure 2: The framework of our proposed method(CMSC-DCCA). $\mathbf{X}_{1}$ and $\mathbf{X}_{2}$ are the input data from two modalities. $\widehat{\mathbf{X}}_{1}$ and $\widehat{\mathbf{X}}_{2}$ are the reconstruct data. $E_{1}$ and $E_{2}$ are deep convolutional encoders. $\mathbf{Z}_{1}$ and $\mathbf{Z}_{2}$ are latent representations from the outputs of $E_{1}$ and $E_{2} . \mathbf{S}$ is the shared coefficient matrix. $D_{1}$ and $D_{2}$ are decoders.

Motivation 1: There are some cross-modal clustering methods based on canonical correlation analysis, such as (Zhang, Zhuang, and Wu 2007; Jin et al. 2015). However, these methods can only learn linear feature. Although some deep cross-modal methods (Wang et al. 2016) have been proposed, they ignores the relationships of the intra-modal data, which cannot well reflect the discriminant information of data. To solve this problem, we propose a cross-modal clustering method based on deep canonical correlation analysis, which can process high-dimensional nonlinear data very well. In order to make full use of the relationships of the inter-modal data and the information of the intra-modal data, we combine the correlation constraint with a self-expression layer to propose the deep cross-modal subspace clustering method (CMSC-DCCA). Assume that the encoded representations for two modalities are defined as $\mathbf{Z}_{1}$ and $\mathbf{Z}_{2}$. The self-expression layer performs the self-expression property for each modality: $\mathbf{Z}_{1}=\mathbf{Z}_{1} \mathbf{S}_{1}$ and $\mathbf{Z}_{2}=\mathbf{Z}_{2} \mathbf{S}_{2}$, where $\mathbf{S}_{1}$ and $\mathbf{S}_{\mathbf{2}}$ are the self-expression coefficient matrices. We maximize the correlations of the inter-modal data $\mathbf{Z}_{1}$ and $\mathbf{Z}_{2}$, whose purpose is to learn a better shared coefficient matrix $\mathbf{S}$ to replace $\mathbf{S}_{1}$ and $\mathbf{S}_{\mathbf{2}}$ to further reflect the structure of two modalities data simultaneously. Our method ensures that different data with high similarities are more likely to be clustered into one group.

Motivation 2: Deep canonical correlation analysis (Deep CCA) (Andrew et al. 2013) solves nonlinear problems of data using deep neural networks. However, it does not consider whether the data encoded by the neural network can maintain the overall structure of the original data. Thus, we add decoders based on Deep CCA, which can reconstruct the latent features from deep canonical correlation analysis model. The decoders aim to ensure that the latent features can reflect the structure of the original data to improve clustering performance.

In this paper, our work not only considers the relationships of the intra-modal data by the self-expression layer, the correlations of the inter-modal data by the correlation constraint, but also preserves the overall data structure by the decoders.

\section{The Framework of CMSC-DCCA}

The proposed Cross-Modal Subspace Clustering via Deep Canonical Correlation Analysis (CMSC-DCCA) method consists of three parts: deep canonical correlation analysis (Deep CCA) model, a self-expressive layer and Deep CCA decoders. The framework is as shown in Figure 2.

Deep CCA Model: In our Deep CCA model, there are two parts: deep convolutional encoders and correlation constraint. We assume that two modalities data are $\mathbf{X}_{1}=$ $\left\{x_{1}^{i}\right\}_{i=1}^{m} \in \mathbb{R}^{d_{1} \times m}, \mathbf{X}_{2}=\left\{x_{2}^{i}\right\}_{i=1}^{m} \in \mathbb{R}^{d_{2} \times m}$, where $m$ is the number of samples. $d_{1}$ and $d_{2}$ are the corresponding dimensions of the modality 1 and the modality 2 . We set deep convolutional encoders to four layers for two modalities. $\mathbf{X}_{1}$ and $\mathbf{X}_{2}$ are fed as inputs to deep convolutional encoders to process and we can obtain the latent representations $\left(\mathbf{Z}_{1} \mid \theta_{e_{1}}\right) \in \mathbb{R}^{o \times m}$ and $\left(\mathbf{Z}_{2} \mid \theta_{e_{2}}\right) \in \mathbb{R}^{o \times m}$, where $\theta_{e_{1}}, \theta_{e_{2}}$ are the parameters of deep convolutional encoders 1 and 2, and $o$ is the output dimension of deep convolutional encoders. Then we calculate the correlations between $\mathbf{Z}_{1}$ and $\mathbf{Z}_{2}$ with the following expression:

$$
\underset{\theta_{e_{1}}, \theta_{e_{2}}}{\arg \max } \operatorname{corr}\left(\mathbf{Z}_{1}, \mathbf{Z}_{2}\right)=\underset{\theta_{e_{1}}, \theta_{e_{2}}}{\arg \max } \frac{\operatorname{cov}\left(\mathbf{Z}_{1}, \mathbf{Z}_{2}\right)}{\sqrt{D\left(\mathbf{Z}_{1}\right)} \sqrt{D\left(\mathbf{Z}_{2}\right)}},
$$

where $\operatorname{corr}(\cdot)$ is the correlation between $\mathbf{Z}_{1}$ and $\mathbf{Z}_{2}$. $\operatorname{cov}\left(\mathbf{Z}_{1}, \mathbf{Z}_{2}\right)$ is the covariance of $\mathbf{Z}_{1}$ and $\mathbf{Z}_{2}$, and $D\left(\mathbf{Z}_{i}\right)$ is the variance of $\mathbf{Z}_{i}, i=1,2$.

Self-expression layer Layer: Some self-expression based methods (Rao et al. 2008; Elhamifar and Vidal 2013; Abavisani and Patel 2018) have received a lot of attention, whose goals are to express the data point as a linear combination of other points in the same subspace. We obtain the latent representations $\mathbf{Z}_{1}$ and $\mathbf{Z}_{2}$ from two modalities 
encoders and send them to the self-expression layer. In the same space, one data point can be represented linearly by other data points. Then we can get the equation:

$$
\mathbf{Z}_{i}=\mathbf{Z}_{i} \mathbf{S}, \quad \text { s.t., } \operatorname{diag}(\mathbf{S})=0,
$$

where $\mathbf{S}$ is the shared self-expression coefficient matrix for two modalities. To avoid the trivial solution $\mathbf{S}=\mathbf{I}$, we constrain $\operatorname{diag}(\mathbf{S})=0$. Then we can leverage the matrix $\mathbf{S}$ to construct the affinity matrix by the following equation:

$$
\mathbf{C}=\frac{1}{2}\left(|\mathbf{S}|+\left|\mathbf{S}^{\top}\right|\right) \text {. }
$$

Finally, we perform spectral clustering on matrix $\mathbf{C}$.

Deep CCA Decoders: The Deep CCA decoder of each modality consists of four-layer neural network: one fully connected layer and three deconvolution decoding layers, which aims to reconstruct the latent representations from the self-expression layer and maintain the structural characteristics of the original data. In our model, the outputs $\mathbf{Z}_{1} \mathbf{S}$ and $\mathbf{Z}_{2} \mathbf{S}$ from the self-expression layer are used as inputs to the two deep CCA decoders. We can obtain the outputs $\left(\widehat{\mathbf{X}}_{1} \mid \theta_{d_{1}}\right)$ and $\left(\widehat{\mathbf{X}}_{1} \mid \theta_{d_{1}}\right)$, where $\theta_{d_{1}}$ and $\theta_{d_{2}}$ are network parameters of the decoders.

\section{Loss Function Analysis}

The goal of the framework is to combine each part to learn a reliable objective function. We give the loss function analysis for each part and the final objective function. Minimize the loss function to optimize our model.

Deep CCA Loss: In the Deep CCA model, we send crossmodal data to deep convolutional encoders and obtain the latent representations $\mathbf{Z}_{1}$ and $\mathbf{Z}_{2}$. We need to maximize the correlations between $\mathbf{Z}_{1}$ and $\mathbf{Z}_{2}$ to improve clustering performance. According to Eq.(1), the goal is to jointly learn parameters for both $\theta_{e_{1}}$ and $\theta_{e_{2}}$. First we centralize data:

$$
\overline{\mathbf{Z}}_{1}=\mathbf{Z}_{1}-\frac{1}{m} \mathbf{Z}_{1} \mathbf{1}, \quad \overline{\mathbf{Z}}_{1}=\mathbf{Z}_{2}-\frac{1}{m} \mathbf{Z}_{2} \mathbf{1} .
$$

According to the calculation method of (Andrew et al. 2013), the optimization goal is:

$$
\arg \max \operatorname{corr}\left(\mathbf{Z}_{1}, \mathbf{Z}_{2}\right)=\arg \max \operatorname{tr}\left(\sqrt{\mathbf{T}^{\top} \mathbf{T}}\right),
$$

where $\mathbf{T}=\widehat{\mathbf{M}}_{11}^{-1 / 2} \widehat{\mathbf{M}}_{12} \widehat{\mathbf{M}}_{22}^{-1 / 2}, \widehat{\mathbf{M}}_{11}=\frac{1}{m-1} \overline{\mathbf{Z}}_{1} \overline{\mathbf{Z}}_{1}^{\top}+$ $r_{1} \mathbf{I}, \widehat{\mathbf{M}}_{22}=\frac{1}{m-1} \overline{\mathbf{Z}}_{2} \overline{\mathbf{Z}}_{2}^{\top}+r_{2} \mathbf{I}$ and $\widehat{\mathbf{M}}_{12}=\frac{1}{m-1} \overline{\mathbf{Z}}_{1} \overline{\mathbf{Z}}_{2}^{\top}$. $r_{1}>0$ and $r_{2}>0$ are the regularization constants, and we set parameters $r_{1}$ and $r_{2}$ as a relatively small value 0.001 to make matrices $\widehat{\mathbf{M}}_{11}$ and $\widehat{\mathbf{M}}_{22}$ inverse. $\operatorname{tr}(\cdot)$ is the trace function of the matrix. Then the final optimization goal for DCCA loss is:

$$
\operatorname{loss}_{\mathrm{DCCA}}=-\min \operatorname{tr}\left(\sqrt{\mathbf{T}^{\top} \mathbf{T}}\right) .
$$

Self-expression Loss: In the self-expression layer, to better perform the self-expression properties and acquire a better self-expression coefficient matrix $\mathbf{S}$, we minimize the self-expression loss function:

$$
\begin{aligned}
\operatorname{loss}_{\mathrm{S}}= & \min \|\mathbf{S}\|_{\mathrm{F}}^{2}+\sum_{i=1}^{2}\left\|\mathbf{Z}_{i}-\mathbf{Z}_{i} \mathbf{S}\right\|_{\mathrm{F}}^{2} \\
& \text { s.t., } \operatorname{diag}(\mathbf{S})=0
\end{aligned}
$$

where $\|\cdot\|_{\mathrm{F}}$ denotes the matrix Frobenius norm.
Reconstruction Loss: In order to guarantee the effectiveness of the representations processed by the Deep CCA encoders and the self-expression layer, we add the Deep CCA decoders to reconstruct data. The representations $\mathbf{Z}_{1} \mathbf{S}$ and $\mathbf{Z}_{2} \mathbf{S}$ from the self-expression layer are fed to the decoders and we can acquire the reconstruct data $\left(\widehat{\mathbf{X}}_{1} \mid \theta_{d_{1}}\right)$ and $\left(\widehat{\mathbf{X}}_{2} \mid \theta_{d_{1}}\right)$. Minimizing errors between reconstructed data and original data can optimize networks. Therefore, the reconstruction loss for the network is:

$$
\operatorname{loss}_{\mathrm{Re}}=\min _{\theta_{d_{1}}, \theta_{d_{2}}} \sum_{i=1}^{2}\left\|\mathbf{X}_{i}-\widehat{\mathbf{X}}_{i}\right\|_{\mathrm{F}}^{2} .
$$

According to analysis of the loss function for each part, the final objective function can be summarized as follows:

$$
\begin{aligned}
\text { Loss }= & \min _{\theta}\|\mathbf{S}\|_{\mathrm{F}}^{2}+\lambda_{1} \sum_{i=1}^{2}\left\|\mathbf{Z}_{i}-\mathbf{Z}_{i} \mathbf{S}\right\|_{\mathrm{F}}^{2}+ \\
& \lambda_{2} \sum_{i=1}^{2}\left\|\mathbf{X}_{i}-\widehat{\mathbf{X}}_{i}\right\|_{\mathrm{F}}^{2}-\lambda_{3} \operatorname{tr}\left(\sqrt{\mathbf{T}^{\top} \mathbf{T}}\right),
\end{aligned}
$$

where $\lambda_{1}, \lambda_{2}, \lambda_{3}>0$ denote regularization parameters. $\|\cdot\|_{F}$ is the matrix Frobenius norm. $\operatorname{tr}(\cdot)$ indicates trace function. $\theta$ is a set of network parameters which includes $\theta_{e_{1}}, \theta_{e_{2}}$ and $\theta_{d_{1}}, \theta_{d_{2}}$.

\section{Train Steps}

In the proposed model, we use two steps to train the model and optimize the network parameters.

First Step: We pre-train the network using Eq.(8). We send the cross-modal data $\mathbf{X}_{1}$ and $\mathbf{X}_{2}$ to deep convolutional encoders $E_{1}$ and $E_{2}$, and obtain the reconstruction data $\widehat{\mathbf{X}}_{1}$ and $\widehat{\mathbf{X}}_{2}$ from the decoders $D_{1}$ and $D_{2}$. In pretraining step, we set the learning-rate to 0.001. Minimize the error between the original data and the reconstruction data to optimize the network and update encoders parameters $\theta_{e_{1}}, \theta_{e_{2}}$ and decoders parameters $\theta_{d_{1}}, \theta_{d_{2}}$, where we use mean squared error (MSE) (Wang and Bovik 2009) to optimize the objective function. MSE is the expected value of the square of the difference between the parameter estimate and the true value of the parameter, which can evaluate the degree of change of data. The smaller the value of the MSE, the better the accuracy of the prediction model describing the experimental data.

Second Step: We train the entire network using Eq.(9), i.e., minimizing the total Loss including deep CCA loss $\operatorname{loss}_{D C C A}$, the self-expression layer loss $\operatorname{loss}_{S}$ and the reconstruction loss loss Re to update model parameters $\theta_{e_{1}}$ and $\theta_{e_{2}}, \theta_{d_{1}}$ and $\theta_{d_{2}}$. We obtain the shared coefficient matrix $\mathbf{S}$ from the self-expression layer, and calculate the affinity matrix $\mathbf{C}=\frac{1}{2}\left(|\mathbf{S}|+|\mathbf{S}|^{\top}\right)$. Finally, we use the affinity matrix $\mathbf{C}$ and spectral clustering method to complete data clustering. The whole training process is summarized as Algorithm 1.

\section{Experiments}

In order to evaluate the performance of our proposed CMSCDCCA model, we conduct the experiments by comparing 


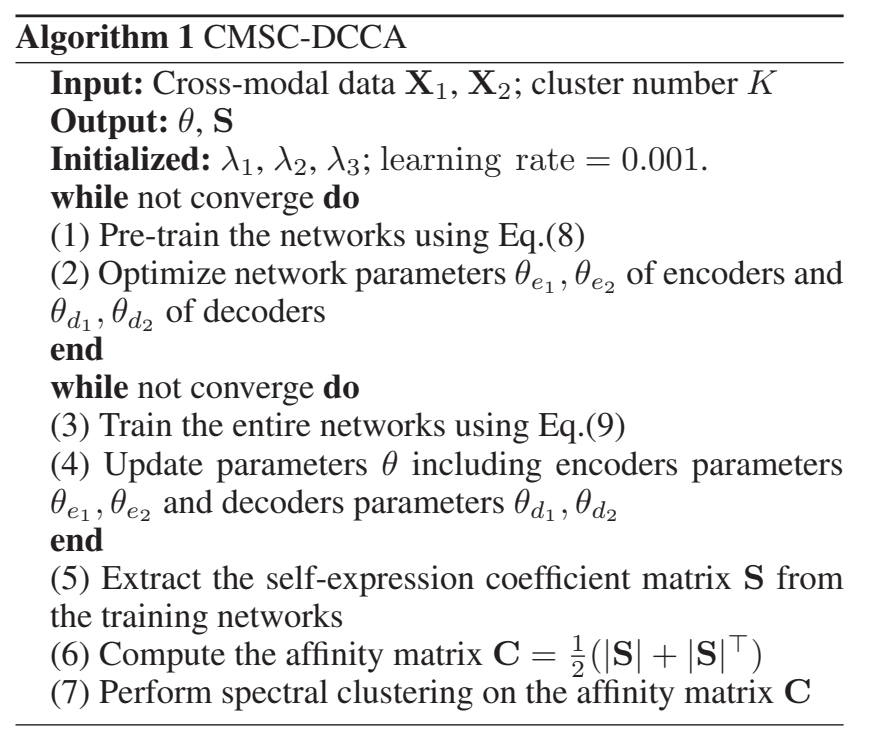

with ten remarkable baseline approaches on four datasets. Specifically, we first introduce four datasets used in our paper, followed by the experiment results and some analysis.

\section{Experiment Setup}

Datasets Settings: The used datasets in our experiments include: 1) FRGC Dataset (Yang, Parikh, and Batra 2016) is an RGB image dataset. In our work, we randomly select 20 objects from the original dataset including 2,462 face images. We set the data size to $32 \times 32$, using its original RGB images as the first modality and its corresponding gray images as the second modality; 2) Fashion-MNIST Dataset (Xiao, Rasul, and Vollgraf 2017) is an image dataset which contains 10 categories with a total of 70,000 different products: t-shirt, shirt, coat, pullover, dress, trouser, bag, sandal, sneaker, ankle boot. The size of each image is $28 \times 28$. In our work, we randomly select 200 samples from per category to make the network easy to handle, and extract their edge features as the second modality; 3) YTF Dataset (Wolf, Hassner, and Maoz 2011) is a face videos dataset which includes 3,425 videos of 1,595 different people. In our work, we select 41 subjects from YTF dataset, and set the size as $55 \times 55$. We use its original RGB images as the first modality, and the gray pictures converted from the original RGB images as the second modality; 4) COIL-20 Dataset consists of 1440 images from 20 objects, and each object includes 72 images that one image is captured for every 5 degrees . In this paper, we use 1440 images and extract their edge features. The 1440 original images and the edge feature images are used as two modalities data.

Implementation details: In our model, we use the fourlayer encoders including three convolution encoding layers and a fully connected layer, and the corresponding decoders consist of a fully connected layer and three deconvolution decoding layers. More specific settings are given in Table 1.

We implement our method and other non-linear methods with the public toolbox of PyTorch. We conduct all the experiments on the platform of Ubuntu Linux 16.04 with
Table 1: The parameters of convolution encoders.

\begin{tabular}{lccc}
\hline Encoders & Convolution kernel size & Stride & Padding \\
\hline Encoder1 & $4 \times 4$ & 2 & 1 \\
Encoder2 & $3 \times 3$ & 1 & 1 \\
Encoder3 & $4 \times 4$ & 2 & 1 \\
\hline
\end{tabular}

NVIDIA Titan Xp Graphics Processing Units (GPUs) and 64 GB memory size. We use Adam (Kingma and Ba 2015) optimizer with default parameter setting to train our model and set the learning rate as 0.001 .

\section{Experimental Results}

Comparison with Existing Approaches: To evaluate the performance of the proposed method, we compare with ten algorithms, including two classic single-modal clustering methods and eight outstanding multi-modal clustering methods. K-means clustering (Hartigan and Wong 1979) and Deep Embedding Clustering (DEC) (Xie, Girshick, and Farhadi 2016) are single-modal clustering methods and are regarded as the baseline algorithms for comparison. Robust Multi-View K-Means Clustering (RMKMC) (Cai, Nie, and Huang 2013) integrates heterogeneous representations of large scale data; Binary Multi-View Clustering (BMVC) (Zhang et al. 2018) dexterously manipulates multi-view image data and easily scales to large data; Joint Framework for Deep Multi-view Clustering (DMJC) (Lin et al. 2018) designs two ingenious variants of deep multiview joint clustering models; Deep Multi-modal Subspace Clustering (DMSC) (Abavisani and Patel 2018) presents a method based on convolutional neural network (CNN) for unsupervised multi-modal subspace clustering; Deep Canonical Correlation Analysis (DCCA) (Andrew et al. 2013) computes correlations between two modalities by deep networks mapping and the correlation constraint; Deep Canonically Correlated Auto-Encoders (DCCAE) (Wang et al. 2016) optimizes the canonical correlations between two learned subspace representations and reconstruct data with the auto-encoders to ensure that the representations are available; Deep Generalized Canonical Correlation Analysis (DGCCA) (Benton et al. 2019) is a method for learning nonlinear transformations of arbitrarily multi-modal data. Cross-Modal Image Clustering via Canonical Correlation Analysis (CMIC-CCA) is an algorithm that supports more effective cross-modal image clustering with Canonical Correlation Analysis (CCA). For DCCA, DCCAE and CMICCCA methods, only two modalities data as inputs can be sent to these models. For RMKMC, BMVC, DMJC, DMSC and DGCCA methods, there are mutli-modal data as inputs to these five models. For comparison, we select two modalities data to do contrast experiments on four datasets.

Performance Evaluation: We adopt two metrics (i.e., clustering accuracy (ACC) (Kuhn 1955), normalized mutual information (NMI) (Xu, Liu, and Gong 2003)) to evaluate the performance by comparing with ten baseline methods on four datasets. The correct clustering should assign the high similarity data to the same group, and different data to dif- 
Table 2: The clustering accuracy rate(ACC)(\%) and the normalized mutual information(NMI)(\%) on four datasets.

\begin{tabular}{|c|c|c|c|c|c|c|c|c|}
\hline \multirow{2}{*}{ Methods } & \multicolumn{2}{|c|}{ Fashion-MNIST } & \multicolumn{2}{|c|}{ COIL-20 } & \multicolumn{2}{|c|}{ FRGC } & \multicolumn{2}{|c|}{ YTF } \\
\hline & $\mathrm{ACC}$ & NMI & $\mathrm{ACC}$ & NMI & $\mathrm{ACC}$ & NMI & $\mathrm{ACC}$ & NMI \\
\hline kmeans & 51.27 & 49.99 & 57.49 & 73.22 & 23.62 & 27.12 & 56.01 & 75.23 \\
\hline $\mathrm{DEC}$ & 51.80 & 54.60 & 68.00 & 80.25 & 37.80 & 50.50 & 37.10 & 44.60 \\
\hline RMKMC & 53.32 & 52.87 & 60.97 & 74.93 & 23.52 & 25.85 & 57.21 & 74.56 \\
\hline BMVC & 45.36 & 38.05 & 34.31 & 40.33 & 41.51 & 45.92 & 28.13 & 38.28 \\
\hline DMJC & 61.41 & 63.41 & 72.99 & 81.58 & 44.07 & 59.79 & 61.15 & 77.40 \\
\hline DMSC & 59.55 & 65.07 & 74.10 & 86.82 & 60.28 & 75.51 & 62.80 & 80.16 \\
\hline DCCA & 52.74 & 53.82 & 55.76 & 64.91 & 22.91 & 24.75 & 45.19 & 60.35 \\
\hline DCCAE & 55.95 & 52.93 & 61.60 & 71.56 & 32.33 & 31.22 & 45.57 & 60.15 \\
\hline DGCCA & 56.28 & 57.04 & 54.01 & 62.40 & 23.76 & 24.53 & 47.26 & 61.38 \\
\hline CMIC-CCA & 53.45 & 51.15 & 59.93 & 72.98 & 31.03 & 34.58 & 59.70 & 76.11 \\
\hline CMSC-DCCA & 62.95 & 68.33 & 82.64 & 91.45 & 70.80 & 78.55 & 66.15 & 82.67 \\
\hline
\end{tabular}

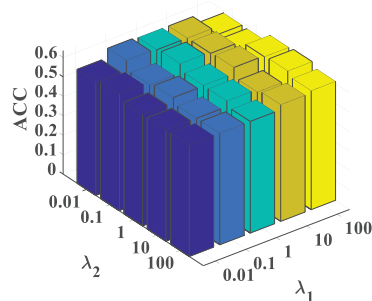

(a)

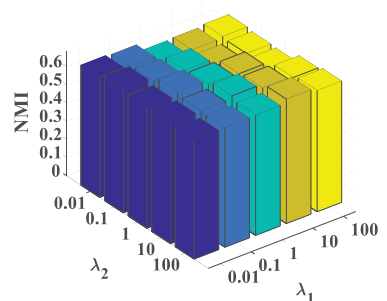

(b)

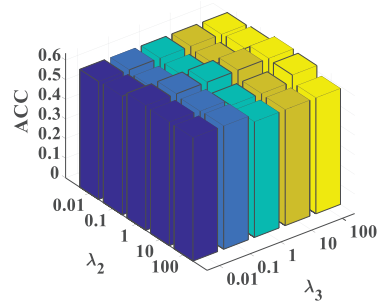

(c)

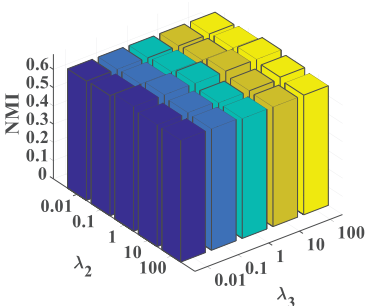

(d)

Figure 3: The effect of parameters $\lambda_{1}, \lambda_{2}$ and $\lambda_{3}$ on Fashion-MNIST dataset, where $\lambda_{1}$ is the regularization parameter of the self-expression error, $\lambda_{2}$ is the regularization parameter of the reconstruction error and $\lambda_{3}$ is the regularization parameter of the correlation calculation. (a) and (b) are the clustering results in terms of ACC and NMI, when fixing $\lambda_{3}$ and varying $\lambda_{1}$ and $\lambda_{2}$. (c) and (d) are clustering results in terms of ACC and NMI, when fixing $\lambda_{1}$, and varying $\lambda_{2}$ and $\lambda_{3}$.

ferent groups. Therefore, the bigger the values of ACC and NMI are, the better the clustering performance of the corresponding method will be.

The clustering performances of our method and comparison algorithms on four datasets are reported in Table 2. From the presented results, we can have the following observations: 1) our proposed CMSC-DCCA model can achieve the best performance on all the four datasets in terms of both ACC and NMI, which verifies the impact of improved clustering performance via the correlations between inter-modal and intra-modal data. 2) Our proposed model significantly outperforms both K-means and DEC among most cases, e.g., on the FRGC dataset, K-means and DEC are only $23.62 \%$ and $37.80 \%$ for ACC, and $27.12 \%$ and $50.50 \%$ for NMI. That is because they are single modality clustering methods, which does not consider information of other modalities. 3) The reason why DCCA obtains poor clustering performance is that it cannot reconstruct data to ensure that the representations after the encoded network can still reflect the structure of the original data; since DCCAE cannot consider the relationships among intra-modal data, which causes ACC and NMI to be $32.33 \%$ and $31.22 \%$ on the FRGC dataset; CMIC-CCA is limited to linear transformations and cannot handle the problems of nonlinear data mapping, resulting in clustering performance low. Additionally, our proposed model also performs better than DMJC and DMSC because these two methods cannot make full use of the correlations among the inter-modal data.

Parameters Analysis: In our model, there are three regularization parameters $\lambda_{1}, \lambda_{2}$ and $\lambda_{3}$ and we simultaneously adjust them to obtain the best model. However, for simplicity and evaluating the effect of each parameter in our experiments, we fix one and vary the other two for each time. Firstly, we fix the regularization parameters of the correlation calculation $\lambda_{3}$, and vary the regularization parameters of the self-expression error and the reconstruction error $\lambda_{1}$ and $\lambda_{2}$ in range $\{0.01,0.1,1,10,100\}$. Then we fix $\lambda_{1}$, and also vary $\lambda_{2}$ and $\lambda_{3}$ in the same range. Since the strategies of setting parameters are the same on all the four datasets, we only show the effect of parameters on Fashion-MNIST dataset for simplicity. From the presented in Figure 3, notice that: 1) our method can achieve the best ACC and NMI values on Fashion-MNIST dataset when $\lambda_{1}=0.1, \lambda_{2}=1$ and $\lambda_{3}=1 ; 2$ ) our method is robust because the changes of parameters have a little influence on the clustering perfor- 


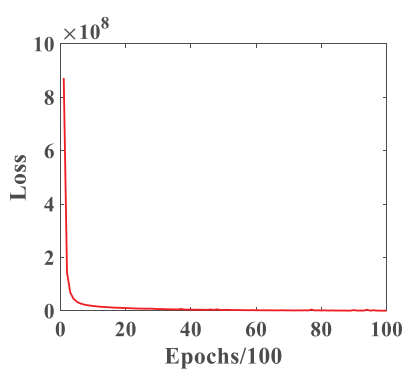

(a) Fashion-MNIST

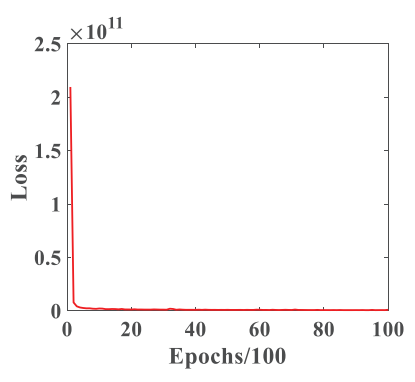

(b) COIL-20

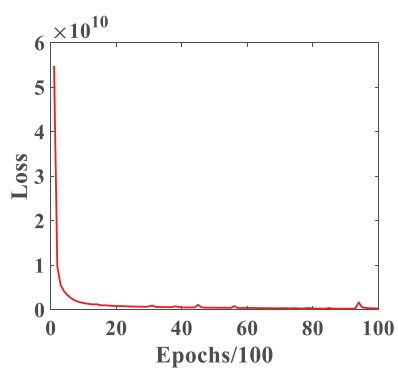

(c) FRGC

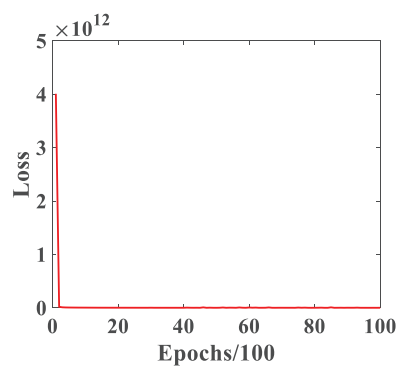

(d) YTF

Figure 4: The loss curves of our method on four datasets, i.e., Fashion-MNIST, COIL-20, FRGC and YTF datasets. We set 10000 epochs to train the entire network and obtain a loss value for each 100 epochs.

mance. But the influence of parameters can not be ignored because they also boost clustering performance to some extent. In addition, the values of best parameters are $\lambda_{1}=1$, $\lambda_{2}=1$ and $\lambda_{3}=1$ for YTF dataset, and are $\lambda_{1}=0.1$, $\lambda_{2}=1$ and $\lambda_{3}=1$ for both FRGC and COIL-20 datasets.

Convergence Analysis: In order to investigate the convergence of our proposed model, in this subsection, we plot the corresponding loss of Eq. (9) on four datasets. As despicted in Figure 4, the values of objective function loss decrease with respect to iterations on all the four datasts, and the values approach to be a fixed value after a few iterations (less than 20 iterations), where each iteration includes 100 epochs. Therefore, our proposed optimization algorithm is reliable and converges quickly.

Through the above analysis, we can find that the clustering performance of our proposed method is highly related to the following aspects: 1) The regularization parameters play a key role in clustering performance. We thus fix the best regularization parameters to train network which aims to learn better network parameters; 2) The clustering performance is closely associated with the number of the pretraining epochs. Appropriate pre-training epochs can improve overall clustering performance; 3) Data preprocessing and initialization methods also have a certain impact on the clustering performance.

Ablation Study: In this subsection, we perform ablation study to analyze the role of each part in our model. For simplicity, we conduct experiments on Fashion-MNIST dataset with different components ablation, i.e., without correlation constraint in deep CCA model, without a self-expressive layer and without deep CCA decoders. As shown in Table 3, we can observe: 1) the correlation constraint has a certain impact on clustering performance, which maximizes the correlations of inter-modal data and obtains a better common subspace representation; 2) the self-expression layer has a significant effect on the proposed model, i.e., the relationships among intra-modal data play an important role in clustering performance; 3) deep CCA decoders have the biggest impact on the proposed method, whose role is to ensure the overall structure of original data and make the encoded data reliable. These above observations indicate that all the three components in our proposed model are designed reasonably.
Table 3: Ablation Study on Fashion-MNIST dataset in terms of ACC (\%) and NMI (\%).

\begin{tabular}{ccc}
\hline Methods & ACC & NMI \\
\hline Without correlation constraint & 60.25 & 61.02 \\
Without self-expression & 55.75 & 56.01 \\
Without decoder & 50.50 & 52.77 \\
CMSC-DCCA & $\mathbf{6 2 . 9 5}$ & $\mathbf{6 8 . 3 3}$ \\
\hline
\end{tabular}

\section{Conclusion}

We propose a novel clustering method named Cross-Modal Subspace Clustering via Deep Canonical Correlation Analysis (CSMC-DCCA). We maximize the correlations of the inter-modal data to make data with high similarities better clustered into the same group by the correlation constraint and make full use of the information of the intra-modal data by the self-expression layer. We construct the shared subspace coefficient matrix based on the self-expression layer. At the same time, we reconstruct the data by the decoders to ensure the overall structure of the original data. Then we optimize the objective function by training the entire network and apply the spectral clustering method to implement clustering. Our experiments demonstrate that the proposed method provides significant improvement over the several state-of-the-art clustering methods.

\section{Acknowledgements}

This work is supported by National Natural Science Foundation of China under Grant 61773302, 61906142, Initiative Postdocs Supporting Program, China Postdoctoral Science Foundation (Grant 2019M653564) and the Fundamental Research Funds for the Central Universities.

\section{References}

Abavisani, M., and Patel, V. M. 2018. Deep multimodal subspace clustering networks. IEEE Journal of Selected Topics in Signal Processing 12(6):1601-1614.

Akaho, S. 2006. A kernel method for canonical correlation analysis. CoRR abs/cs/0609071. 
Akata, Z.; Thurau, C.; and Bauckhage, C. 2011. Nonnegative matrix factorization in multimodality data for segmentation and label prediction. $C V W W$.

Andrew, G.; Arora, R.; Bilmes, J. A.; and Livescu, K. 2013. Deep canonical correlation analysis. In ICML, 1247-1255.

Benton, A.; Khayrallah, H.; Gujral, B.; Reisinger, D. A.; Zhang, S.; and Arora, R. 2019. Deep generalized canonical correlation analysis. In Proceedings of the Workshop on Representation Learning for NLP, 1-6.

Cai, X.; Nie, F.; and Huang, H. 2013. Multi-view k-means clustering on big data. In IJCAI, 2598-2604.

Chaudhuri, K.; Kakade, S. M.; Livescu, K.; and Sridharan, K. 2009. Multi-view clustering via canonical correlation analysis. In ICML, 129-136.

Elhamifar, E., and Vidal, R. 2013. Sparse subspace clustering: Algorithm, theory, and applications. TPAMI 35(11):2765-2781.

Guo, D.; Zhang, J.; Liu, X.; Cui, Y.; and Zhao, C. 2014. Multiple kernel learning based multi-view spectral clustering. In ICPR, 3774-3779.

Hartigan, J. A., and Wong, M. A. 1979. Algorithm as 136: A k-means clustering algorithm. Journal of the Royal Statistical Society 28(1):100-108.

He, R.; Zhang, M.; Wang, L.; Ji, Y.; and Yin, Q. 2015. Cross-modal subspace learning via pairwise constraints. TIP 24(12):5543-5556.

Ji, P.; Zhang, T.; Li, H.; Salzmann, M.; and Reid, I. D. 2017. Deep subspace clustering networks. In NeurIPS, 24-33.

Jia, Y.; Salzmann, M.; and Darrell, T. 2011. Learning crossmodality similarity for multinomial data. In ICCV, 24072414.

Jin, C.; Mao, W.; Zhang, R.; Zhang, Y.; and Xue, X. 2015. Cross-modal image clustering via canonical correlation analysis. In $A A A I, 151-159$.

Kim, T.; Kittler, J.; and Cipolla, R. 2007. Discriminative learning and recognition of image set classes using canonical correlations. TPAMI 29(6):1005-1018.

Kingma, D. P., and Ba, J. L. 2015. Adam: A method for stochastic optimization. In ICLR.

Kuhn, H. W. 1955. The hungarian method for the assignment problem. Naval Research Logistics Quarterly 2(1):8397.

Li, Z.; Wang, Q.; Tao, Z.; Gao, Q.; and Yang, Z. 2019. Deep adversarial multi-view clustering network. In IJCAI, 29522958.

Lin, B.; Xie, Y.; Qu, Y.; and Li, C. 2018. Deep multi-view clustering via multiple embedding. CoRR abs/1808.06220.

Liu, W., and Tsang, I. W. 2017. Making decision trees feasible in ultrahigh feature and label dimensions. Journal of Machine Learning Research 18:81:1-81:36.

Liu, W.; Tsang, I. W.; and Müller, K. 2017. An easy-to-hard learning paradigm for multiple classes and multiple labels. Journal of Machine Learning Research 18:94:1-94:38.
Nie, F.; Cai, G.; and Li, X. 2017. Multi-view clustering and semi-supervised classification with adaptive neighbours. In AAAI, 2408-2414.

Patel, V. M.; Van Nguyen, H.; and Vidal, R. 2015. Latent space sparse and low-rank subspace clustering. IEEE J-STSP 9(4):691-701.

Rao, S. R.; Tron, R.; Vidal, R.; and Ma, Y. 2008. Motion segmentation via robust subspace separation in the presence of outlying, incomplete, or corrupted trajectories. In $C V P R$, $1-8$.

Rasiwasia, N.; Pereira, J. C.; Coviello, E.; Doyle, G.; Lanckriet, G. R.; Levy, R.; and Vasconcelos, N. 2010. A new approach to cross-modal multimedia retrieval. In ACM MM, 251-260.

Sun, T., and Chen, S. 2007. Locality preserving cca with applications to data visualization and pose estimation. Image and Vision Computing 25(5):531-543.

Sun, G.; Cong, Y.; Li, J.; and Fu, Y. 2018. Robust lifelong multi-task multi-view representation learning. In ICBK, 9198.

Wang, Z., and Bovik, A. C. 2009. Mean squared error: Love it or leave it? a new look at signal fidelity measures. IEEE 26(1):98-117.

Wang, W.; Arora, R.; Livescu, K.; and Bilmes, J. A. 2016. On deep multi-view representation learning: Objectives and optimization. CoRR abs/1602.01024.

Wolf, L.; Hassner, T.; and Maoz, I. 2011. Face recognition in unconstrained videos with matched background similarity. In $C V P R, 529-534$.

Xia, R.; Pan, Y.; Du, L.; and Yin, J. 2014. Robust multi-view spectral clustering via low-rank and sparse decomposition. In $A A A I, 2149-2155$.

Xiao, H.; Rasul, K.; and Vollgraf, R. 2017. Fashion-mnist: a novel image dataset for benchmarking machine learning algorithms. CoRR abs/1708.07747.

Xie, J.; Girshick, R.; and Farhadi, A. 2016. Unsupervised deep embedding for clustering analysis. In ICML, 478-487.

Xu, W.; Liu, X.; and Gong, Y. 2003. Document clustering based on non-negative matrix factorization. In ACM SIGIR, 267-273.

Yang, J.; Parikh, D.; and Batra, D. 2016. Joint unsupervised learning of deep representations and image clusters. In $C V P R, 5147-5156$.

Yin, Q.; Wu, S.; He, R.; and Wang, L. 2015. Multi-view clustering via pairwise sparse subspace representation. Neurocomputing 156:12-21.

Zhang, Z.; Liu, L.; Shen, F.; Shen, H. T.; and Shao, L. 2018. Binary multi-view clustering. TPAMI 41(7):1774-1782.

Zhang, H.; Zhuang, Y.; and Wu, F. 2007. Cross-modal correlation learning for clustering on image-audio dataset. In ACM MM, 273-276. 